\title{
Etudes préliminaires sur les ouvrages de prise d'eau pour une centrale nucléaire en bordure de la Méditerranée
}

\author{
PAR
}

R. Lacroix

Président de S.F.P.-Structures

\author{
E. Collange \\ Ingénieur en chef S.G.E.
}

ET

\author{
C. Lepère \\ Ingénieur en chef S.F.P.-Structures
}

Il paraît au départ nécessaire de souligner deux points :

- Le fait que les études prospectives faites doivent beaucoup, d'une part à la Direction de l'Equipement de l'Electricité de France qui a parrainé l'étude, d'autre part aux Services de la Région d'Equipement de Marseille qui l'ont suivie et conseillée dans son développement.

- Le caractère très prospectif des ouvrages de prise d'eau présentés qui les différencie de ceux des solutions qui viennent d'être traitées. Incité à faire preuve d’imagination, le conférencier se sent obligé, au départ, de demander l'indulgence de l'assemblée pour tous les problèmes incomplètement résolus et qui ne passeront pas inaperçus aux membres de cette assemblée qui les connaissent bien : souvent, le cadre de cette conférence obligera à les passer sous silence.

\section{Objet de l'étude}

Nous savons tous que le débit nécessaire aux circuits de refroidissement des centrales nucléaires est important $44 \mathrm{~m}^{3 /} \mathrm{s}$ pour une élévation de température de $14^{\prime \prime}$ ou 15 " $\mathrm{C}$ par tranche de $1350 \mathrm{MW}$, soit $176 \mathrm{~m}^{3 / \mathrm{s}}$ pour quatre tranches - débit encore augmenté s'il devient nécessaire de limiter davantage la température de l'eau restituée.

Dans le cas présent, cette eau est à prendre à partir d'une côte sableuse de mer pratiquement sans marée : amplitude de marnage observée: $+0,80-0,42$ soit $+1,00-0,50$ compte tenu de l'incidence des courants.

Afin de donner un support concret à l'étude, deux implantations ont été considérées :

- l'implantation au site de Port-la-Nouvelle, déjà retenue, aux pentes très douces (inférieures à $1 \%$ );
- l'implantation au site de l'anse des Paulilles, dont la pente moyenne des fonds est voisine de $1,8 \%$.

Concrètement. la prise d'eau est étudiée pour un fond de - 15,00 pour le premier site; la profondeur correspondante est très voisine du minimum pour l'installation d'une station de pompage de cette importance, dans le cas présent elle est atteinte à environ $1850 \mathrm{~m}$ du rivage.

Le deuxième site a été choisi à dessein avec une pente plus rapide, afin d'y étudier une prise d'eau plus profonde : la profondeur de prise a été fixée a priori à - 40 . profondeur qu'on trouve à $2200 \mathrm{~m}$ du rivage.

On s’est efforcé d'apprécier l'incidence des variations des données précédentes. tant en ce qui concerne la profondeur de prise d'eau que la pente du rivage. en tâchant de situer les valeurs critiques de la profondeur qui entraîneraient un changement de solution (difficultés d'exécution et variation brusque du coût).

Les autres paramètres pris en considération sont les suivants :

- Houle: La houle maximale considérée pour les deux sites correspond à une double amplitude maximale de $6 \mathrm{~m}$, observée en 1965. une vague de cette amplitude restant isolée. Période significative de la houle irréguliere correspondante voisine de 10 secondes.

- Vent : Force $7.200 \mathrm{~kg} / \mathrm{m}^{2}$ en exploitation.

- Courants : Essentiellement côtiers et complexes.

Devant, au départ. examiner les autres possibilités que celles des solutions traitées par les conférenciers précédents, il n’a été considéré que deux types de solutions possibles :

- conduites immergées en mer;

- conduites en aqueduc. 


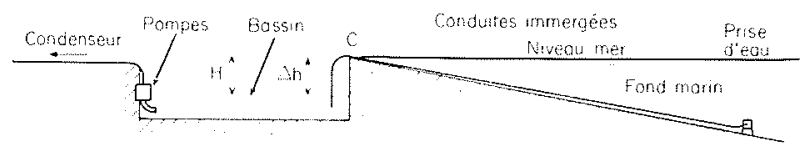

1 / Schéma A

\section{Amenée d'eaul par conduites immergées}

Un schéma type unique, représenté sur la figure 1, figure les différentes parties de l'amenée d'eau:

- une prise d'eau en mer aussi rudimentaire que possible;

- des conduites immergées pour le transport en mer du débit;

- un bassin à terre pour l'alimentation des groupes de pompage : ce bassin s'allonge en devenant un long chenal si la centrale est assez loin du rivage, comme au site de Port-la-Nouvelle.

Le bassin est alimenté en fonction de la dénivellation $\Delta h$ entre le niveau moyen de la mer et le niveau d'eau du bassin. $\Delta h$ est égal, en régime permanent, à la somme des pertes de charge de prise d'eau et de la conduite. La présence d'un coude en $C$ ne s'impose que pour les valeurs importantes de $\Delta h$.

La hauteur $H$ est limitée de façon à ce que la dépression en régime permanent, à plein débit et dans le cas où les dépôts incrustés sur les conduites sont les plus importants. ne dépasse pas $7,50 \mathrm{~m}$ (soit une dépression absolue supérieure à 2,50 $\mathrm{m}$ de colonne d'eau). Rien ne s'oppose à ce que le point haut de la conduite soit au-dessous du niveau d'eau du bassin : on évite ainsi le coude des conduites moyennant un approfondissement de la conduite près de la rive.

\section{RAISONS D'UN CHOIX UNIQUE}

- Une première constatation est le fait que l'amenée par conduites immergées est, indépendamment de la solu- tion "amenée par canal ". la seule solution qui permette le maintien d'une station de pompage terrestre : en effet, tout siphonage par conduites aériennes de grandes dimensions conduit à des dépressions incompatibles avec leur fonctionnement et le transport par conduites flottantes a été exclu au départ (vulnérabilité, obstacle à la navigation).

La préférence des exploitants allant à une station de pompage terrestre. I'utilisation de conduites immergées avec station de pompage en mer, ne semble à être envisagé que dans des cas très exceptionnels (cas du schéma $B^{\prime \prime}$ de la figure 2). En solution A, la prise d'eau en mer sera conçue effectivement de façon à limiter au maximum les interventions: éliminations des éléments électromécaniques de fermeture.

- La deuxième constatation faite est que tout transport terrestre de gros débit est plus économique par canal que par conduite: sauf contraintes particulières, le trajet terrestre est à concevoir par canal plutôt que par conduites enterrées: le canal se situe logiquement à l'amont des pompes et permet, en rapprochant la station de pompage et la centrale nucléaire, de réduire au maximum lá longueur des conduites. L'éloignement de la station de pompage de la centrale pourrait cependant être envisagé. en particulier pour des pompes alimentées directement par conduites d'aspiration, sans bassin intermédiaire de reprise; un autre mode de transport que les conduites reste possible entre la station de pompage et la centrale nucléaire: la réalisation d'un canal surélevé dont le plan d'eau a une charge suffisante pour permettre la circulation de l'eau dans les circuits de refroidissement (cas du schéma C).

De telles conceptions peuvent éviter de disposer la station de pompage en eaux profondes.

\section{NOMBRE DE CONDUITES}

Il convient de remarquer que les débits unitaires des conduites ne sont pas liés à celui des pompes, l'existence d'un bassin de reprise assez étendu rend les circuits de pompe indépendants, de sorte que le nombre des conduites est relativement arbitraire.

Les vitesses de circulation correspondant aux systèmes

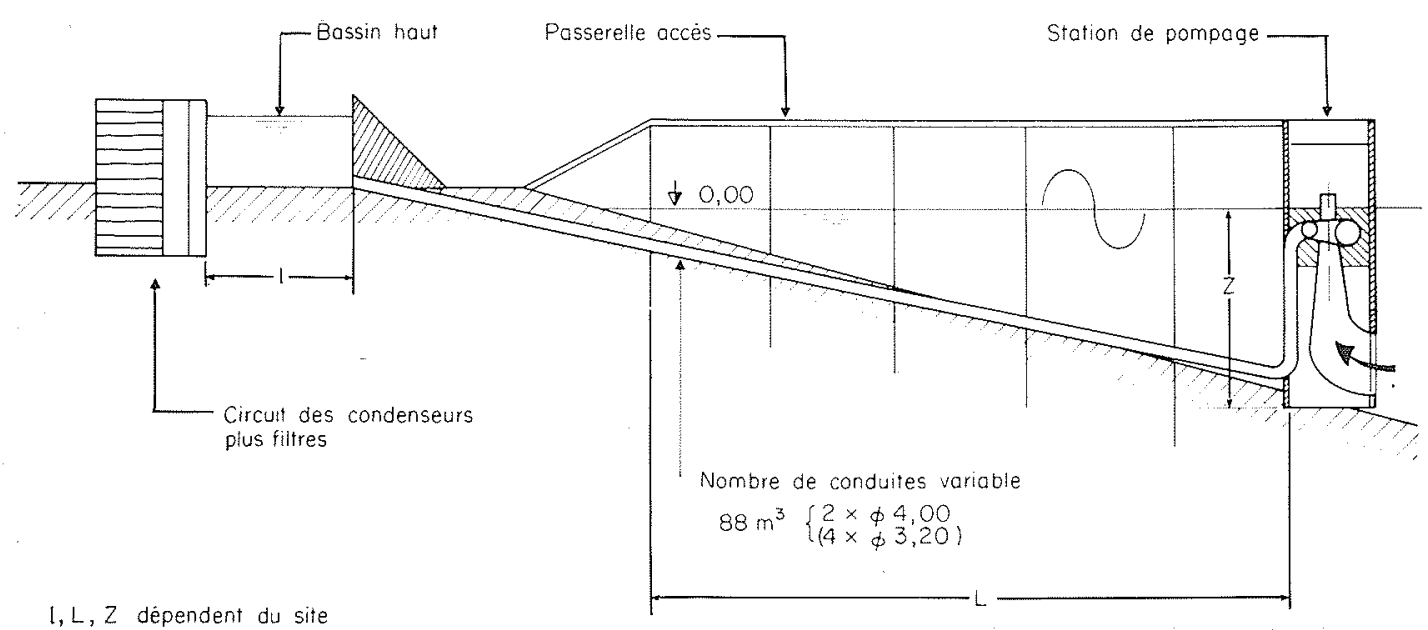

2 / Schéma B" 


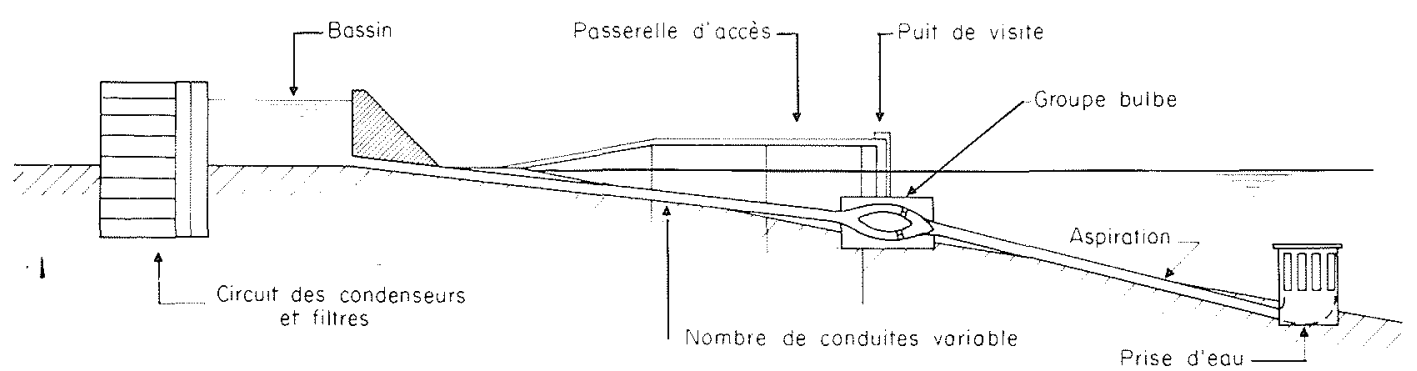

3 / Schéma C

\begin{tabular}{|c|c|c|c|c|c|c|c|}
\hline \multicolumn{8}{|c|}{ Tableau 1} \\
\hline \multicolumn{8}{|c|}{$\begin{array}{l}\text { VITESSES DE CIRCLLATION } \\
(\mathrm{m} / \mathrm{s})\end{array}$} \\
\hline $\begin{array}{l}\text { Diamètres ...(m) } \\
\text { Sections ... }\left(\mathrm{m}^{2}\right)\end{array}$ & $\begin{array}{l}2.75 \\
5.94\end{array}$ & $\left|\begin{array}{l}2.90 \\
6.60\end{array}\right|$ & $\begin{array}{l}3.20 \\
8.04\end{array}$ & $\begin{array}{l}3.50 \\
9.62\end{array}$ & $\begin{array}{r}3.80 \\
11.34\end{array}$ & \begin{tabular}{|c|}
4 \\
12.57
\end{tabular} & $\begin{array}{r}4.20 \\
13.85\end{array}$ \\
\hline $\begin{array}{l}8 \text { conduites ... } \\
6 \text { conduites ... } \\
5 \text { conduites ... } \\
4 \text { conduites ... } \\
3 \text { conduites ... }\end{array}$ & 3.70 & 3.33 & $\begin{array}{l}3.73 \\
3.65\end{array}$ & $\mid \begin{array}{l}2.28 \\
3.05 \\
3.66\end{array}$ & $\begin{array}{l}2.59 \\
3.10 \\
3.88\end{array}$ & 3.50 & $\begin{array}{l}3.18 \\
4.30\end{array}$ \\
\hline
\end{tabular}

étudiés figurent dans le tableau 1 pour le débit global considéré de $176 \mathrm{~m}^{3 / 3}$.

- La réalisation en deux conduites n'a pas été retenue: elle exige lutilisation de tuyaux de plus gros diametre, pour lesquels la mise en place en mer est une extrapolation plus large des réalisations antérieures et conduit les constructeurs trop loin au-dela de leur champ d’expérience actuel. Par ailleurs, lorsque la centrale est limitée a deux tranches, elle aurait à fonctionner avec une seule conduite d"alimentation, ce qui ne parait pas acceptable compte tenu de la nécessité éventuelle d'intervention pour nettoyage.

\section{ENTRETIEN ET CHOIX DES TUYAUX}

Le choix du type de tuyaux est lié à une option fondamentale : l'entretien des conduites.

Ce problème peut revêtir une importance déterminante : l'économie du projet est très différente suivant que l'entretien des conduites nécessite ou non leur vidange.

En effet, les conduites doivent être suffisamment lestées ou chargées pour leur permettre de résister aux efforts de soulevement dus à la houle dans un milieu où le sable est mis en émulsion. Ce poids devient relativement important si on doit envisager que les conduites puissent être mise hors de l'eau pour entretien.

Dans le tableau 2, dans lequel tous les poids sont en T/mètre linéaire de conduite. le poids minimum pour la stabilité est calculé en fonction d'une poussée correspondant à un liquide de densité fictive 1.5. On voit que la stabilité conduite pleine d'eau est déjà mal assurée pour les diametres de $3.50 \mathrm{~m}$ et au-dessus (comparaison des colonnes 4 et 6). L'obtention de la stabilité, en cas de vidange. nécessite de charger les conduites avec des lests correspondants a la colonne 7 . ou de prévoir un ensouillement offrant une garantie équivalente de l'ordre de $4 \mathrm{~m}$ pour une conduite de $03.50 \mathrm{~m}$ soit une profondeur de souille de $8 \mathrm{~m}$ avant mise en place).

Différentes possibilités efficaces de curage des conduites existent et sont actuellement appliquées: le curage mécanique avec racleuses rotatives est le plus efficace pour les conduites de gros diamètre. La "marinisation" des machines de nature à effectuer ce travail est possible: une Société telle que la Société Comex est en mesure de mettre au point de telles machines, le problème est de bien définir les conditions d'utilisation.

\begin{tabular}{|c|c|c|c|c|c|c|}
\hline \multicolumn{7}{|c|}{ Tableau 2} \\
\hline 1 & Epaisseur & $\begin{array}{l}3 \\
\text { Poids } \\
\text { dans } \\
\text { l'air }\end{array}$ & $\begin{array}{c}4 \\
\text { Poids } \\
\text { de la conduite } \\
\text { pleine deau }\end{array}$ & $\begin{array}{l}5 \\
\text { Poussee dans } \\
\text { leau de mer } \\
d=1,03\end{array}$ & $\begin{array}{c}6 \\
\text { Poids minimum } \\
\text { pour là } \\
\text { stabilité }\end{array}$ & $\begin{array}{l}7 \\
\text { Poids } \\
\text { at ajouler } \\
\text { at vide }\end{array}$ \\
\hline $\begin{array}{l}3,20 \mathrm{~m} \\
3,50 \mathrm{~m} \\
4,50 \mathrm{~m}\end{array}$ & $\begin{array}{l}0,28 \mathrm{~m} \\
0.31 \mathrm{~m} \\
0,40 \mathrm{~m}\end{array}$ & $\begin{array}{r}7.7 \\
9.3 \\
15.4\end{array}$ & $\begin{array}{l}16.0 \\
19.2 \\
31.8\end{array}$ & $\begin{array}{r}9.6 \mathrm{~T} \\
13.7 \mathrm{~T} \\
22.7 \mathrm{~T}\end{array}$ & $\begin{array}{l}14.0 \\
19.9 \\
33.0\end{array}$ & $\begin{array}{r}6.7 \\
10.6 \\
17.6\end{array}$ \\
\hline
\end{tabular}


On s'est donc limité à l'étude de l'exécution et de la mise en place de conduites dont la vidange ultérieure serait totalement exclue. Notons que l'option retenue permet, en outre, de ne pas exiger une étanchéité totale des conduites.

On a considéré cependant que les visites d'inspection ou de nettoyage seraient exécutées avec un courant d'eau nul ou très faible: un batardage des conduites est donc à prévoir côté bassin et un puits d'accès, pour accéder aux conduites, après pose des batardeaux.

Malgré cela, un certain ensouillement est indispensable pour les faibles profondeurs, l'ensouillement a été pris égal à $2.50 \mathrm{~m}$, il diminue vers le large et s'annule au voisinage de la prise d'eau.

Compte tenu du poids et des diamètres nécessaires, on s'est limité aux conduites en béton. Pour leur fabrication et pose. contact a été pris avec la Société Bonna du fait de son expérience antérieure.

Les tuyaux âme en tôle et double revêtement en béton armé ont été retenus pour les conduites immergées, de préférence à des tuyaux à simple emboîtement : l'âme en tôle empêche. en effet, la dissociation des éléments lors du transport en mer et après immersion. L'utilisation de tuyaux moins chers n'est toutefois pas définitivement à exclure, compte tenu de l'option prise pour l'entretien des conduites et de la non-nécessité d'une étanchéité totale: une étude plus poussée serait toutefois à faire, portant en particulier sur les joints, pour permettre de conclure à l'intérêt de leur utilisation.

\section{POSE DES CONDUITES}

Le problème de la pose des conduites a été étudié en liaison avec la Société Bonna, elle-même en relation avec la Société Wisser \& Smit.

Deux modes de pose peuvent être envisagés pour les conduites de grande rigidité telles que prévues:

1" une méthode par tirage sur le fond, telle que celle utilisée pour l'émissaire de Cannes:

2" une méthode par assemblage de tronçons importants à terre, transport par flottaison, immersion et raccordement en fond de mer avec l'élément précédent.

Nous mentionnerons pour mémoire la méthode par flottaison et raccordement réalisé. avant immersion, à partir d'une embarcation. Cette méthode, utilisée sur les Sea line métalliques, n’est évidemment pas applicable aux conduites de limportance de celles envisagées ici.

La figure 4.1, qui reproduit des croquis figurant dans "L étude des émissaires marins " de J.-P. Lepetit. du Laboratoire National Hydraulique d’E.D.F., montre le principe de la première méthode utilisée pour l'émissaire de Cannes.

La figure 4.2 montre, schématiquement, le principe de la deuxième méthode qui a été retenue dans le cas présent.

La souille doit être exécutée peu de temps avant l'amenée des éléments concernés sur une longueur convenable.

Les tuyaux sont fabriqués dans une usine de préfabrication réalisée sur le chantier mème. ils sont ensuite assemblés en tronçons plus longs (par exemple longs de $48 \mathrm{~m}$. obtenus en assemblant 8 tuyaux de $6 \mathrm{~m}$ par joints soudés). Les tronçons, assemblés parallèlement au rivage. sont ensuite mis à l'eau et flottés : un ponton, constitué de deux barges flottantes jumelées, accueille les tronçons et les

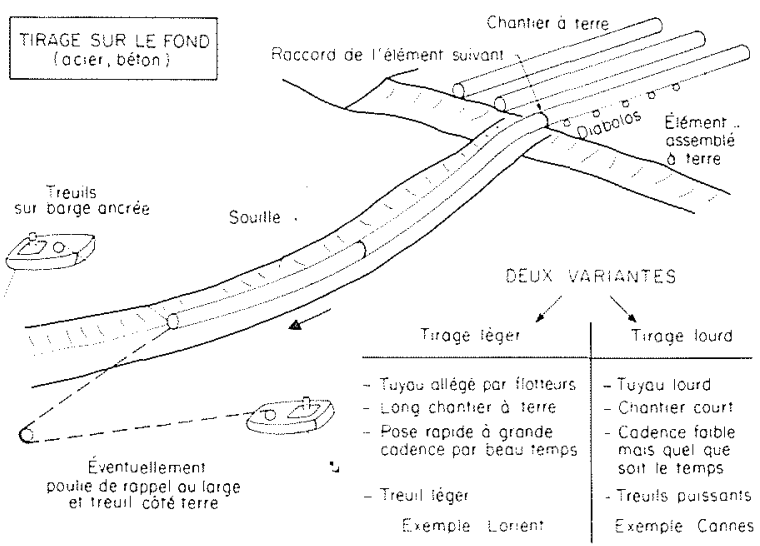

4.1 / Princlpaux modes de pose (tirage)

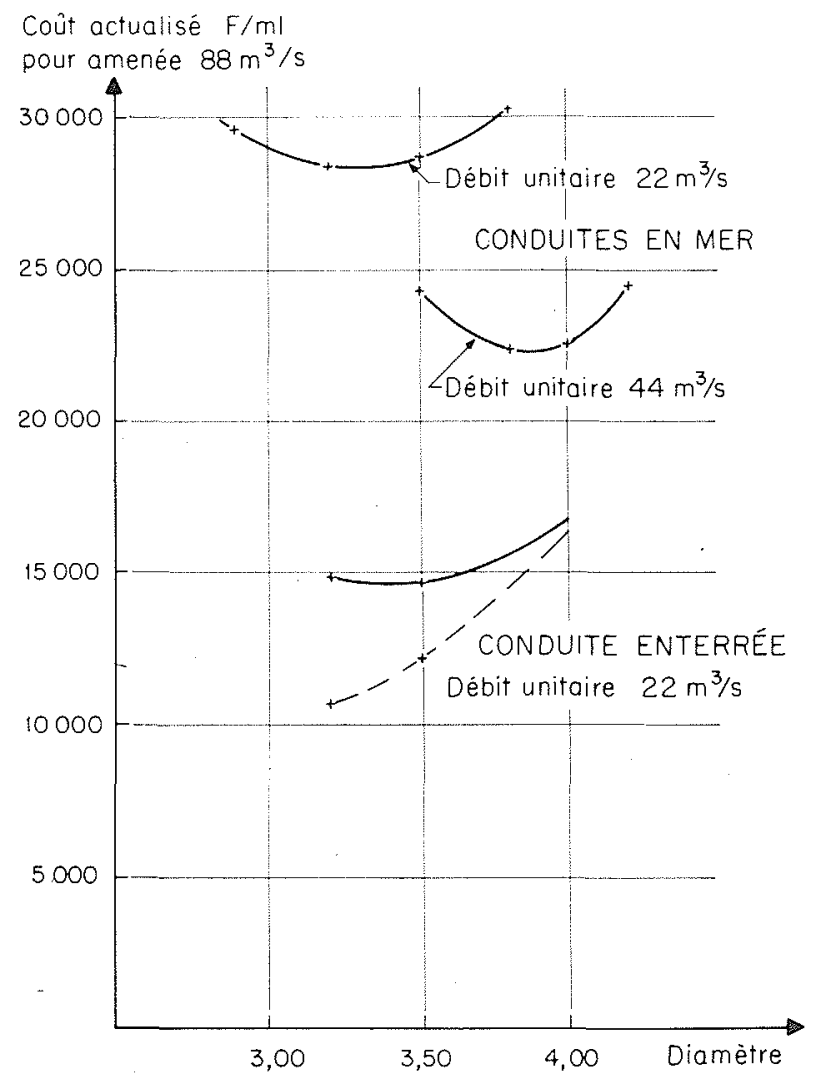

5 / Dlamètre économique

Tableau 3

DIAMETRES ECONOMIQUES

\begin{tabular}{|c|c|c|}
\hline $\begin{array}{c}\text { Débit } \\
\left(\mathrm{m}^{3} / \mathrm{s}\right)\end{array}$ & $\begin{array}{c}\text { Tuyaux } \\
\text { à âme tôle }\end{array}$ & $\begin{array}{c}\text { Tuyaux MVR } \\
\text { sans âme }\end{array}$ \\
\hline 22 & 3,20 & 3,40 \\
35 & 3,60 & - \\
44 & 3,85 & - \\
\hline
\end{tabular}




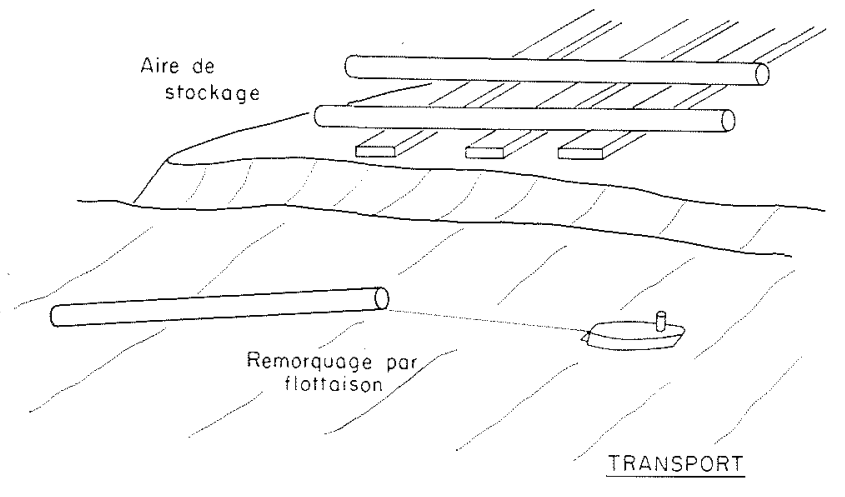

4.2 / Pose par barges fllottantes

guide jusqu'au module muni de vérins qui, après descente au fond, positionne l'élément de conduite.

\section{CARACTÉRISTIQUES ÉCONOMIQUES DES CONDUITES}

Dans l'hypothèse où le bassin faisant suite aux conduites est court, l'incidence de son approfondissement est négligeable devant les variations du coût des conduites en fonction de leur section. Le diamètre économique peut alors être déterminé, indépendamment des longueurs des ouvrages, en rendant minimum le coût actualisé du mètre linéaire de conduite:

$$
A=P+(\rho g Q J a) / 0,65
$$

$P=$ coût du mètre de conduite fourni et posé;

$J=$ perte de charge du mètre linéaire (majorés de $10 \%$ pour pertes de charge singulières proportionnelles);

$Q \quad$ = débit de la conduite (fonctionnement normal);

$0,65=$ rendement admis de l'installation de pompage;

$a=$ coût actualisé du $\mathrm{kW}$, charges de combustibles comprises.

L'incidence de l'augmentation de puissance sur le coût du matériel électromécanique est négligée.

La variation rapide de certains prix, à l'heure actuelle, peut modifier la valeur relative de $P$ et $a$ : pour tenir compte de cette incertitude, il est possible de faire varier les hypothèses prises pour les calculs.

Les diamètres économiques ainsi déterminés restent pratiquement les mêmes dans d'assez larges limites de variations de $P$ et $a$. Les principaux résultats sont condensés dans la figure 5 . correspondant à une des hypothèses considérées : elle fait apparaître les diamètres économiques indiqués dans le tableau 3

\section{Incidence des dragages}

Elle est faible sur l'économie du système et ne modifie pas les résultats.

\section{Incidence de la profondeur de prise d'eau}

Elle contribue à abaisser le diamètre économique mais pas de façon sensible.

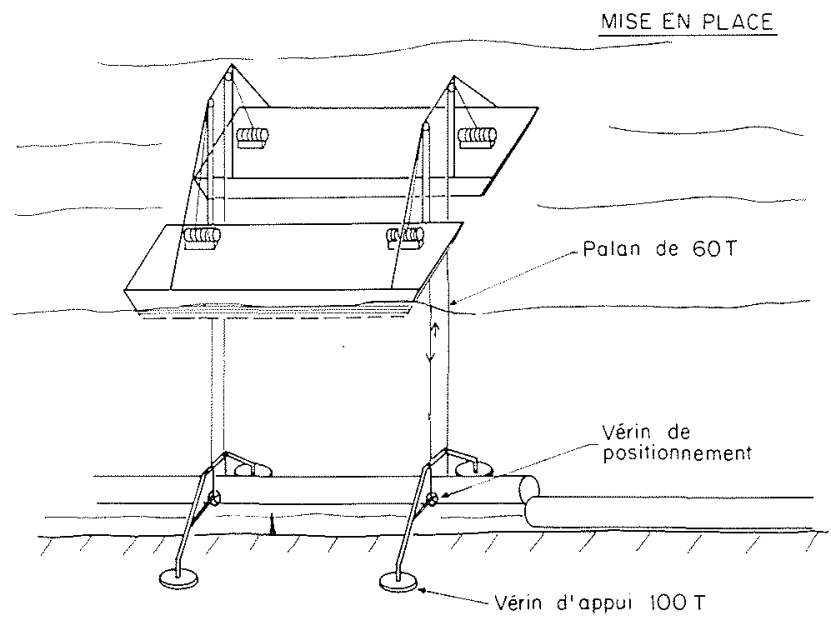

Incidence de l'approfondissement de bassin

Lorsque la centrale est éloignée du rivage (site de Portla-Nouvelle), le coût relatif du bassin, par rapport à celui de l'ensemble des conduites descendant à $-15,00$, est de l'ordre de $20 \%$; l'incidence de son approfondissement n'a qu'un effet limité : il justifie sensiblement le choix d'un diamètre de $4 \mathrm{~m}$ pour le débit de $44 \mathrm{~m}^{3} / \mathrm{s}$ plutôt que 3,80 ; la différence d'approfondissement du bassin étant de $1,40 \mathrm{~m}$.

\section{Construction en deux étapes}

Le fait de pouvoir différer la fourniture et pose des conduites concernant les deuxième et troisième tranches nucléaires est important.

L'abaissement du coût actualisé en différant la dépense $D$ atteint en effet :

$0,317 \mathrm{D}$ pour un décalage de 4 ans

$0,380 \mathrm{D}$ pour un décalage de 5 ans (taux d'actualisation $10 \%$ )

Ces coefficients justifient pleinement l'exécution du chantier des conduites en deux phases distinctes; ils ne justifient pas cependant l'exécution de deux canaux jumelés si ce dédoublement n'est pas justifié par d'autres considérations.

\section{AUTRES OUVRAGES}

Les conduites constituent l'élément essentiel et caractéristique de l'aménagement : nous passerons sous silence la station de pompage qui n'a pas de caractéristiques particulières, en nous bornant à quelques considérations portant sur les ouvrages annexes :

\section{Bassin ou chenal reliant la station de pompage et les conduites}

Le bassin doit fournir un volant d'eau nécessaire entre les conduites et la station de pompage pour absorber des variations rapides de débit; en fait, le bassin aurait été constitué, dans le cas de Port-la-Nouvelle, par le chenal de transport de l'eau depuis la sortie des tuyaux jusqu'à l'en- 


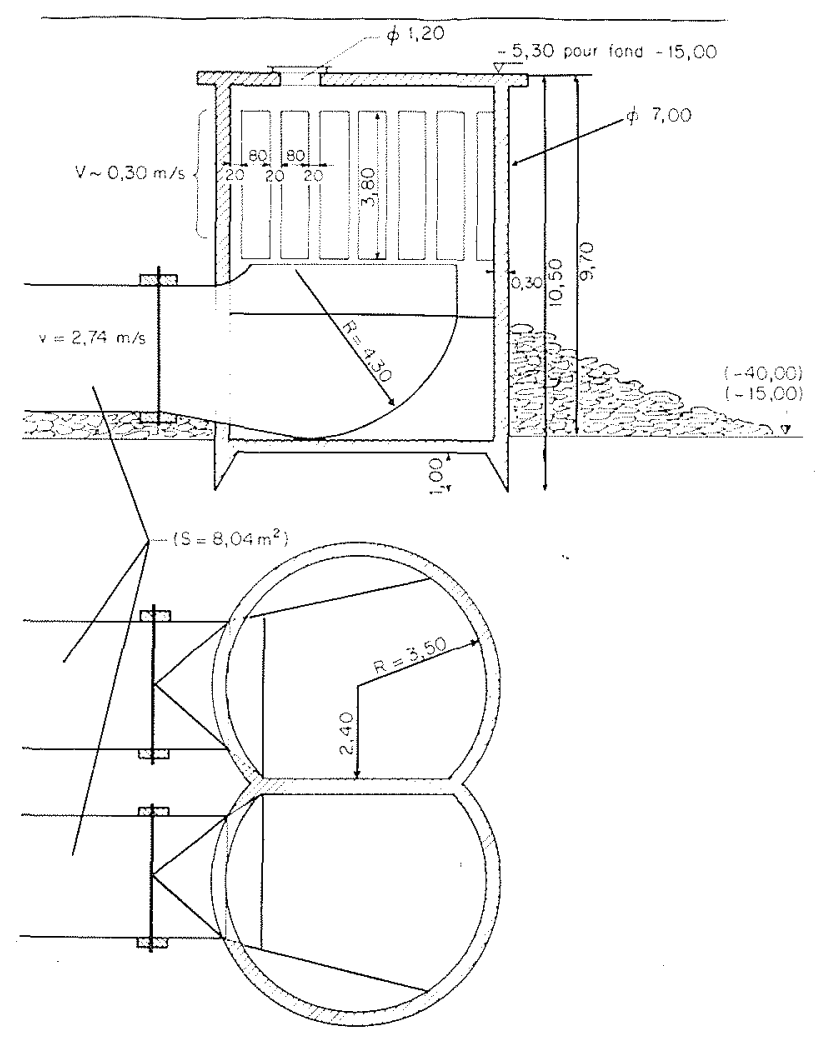

6 / Prlse d'eau

trée des amenées d'eau aux filtres de la station de pompage.

La cote du plan d'eau normal du bassin est fonction des caractéristiques des conduites : pour les sections économiques retenues $\emptyset 4.00$. débit unitaire $44 \mathrm{~m}^{3} / \mathrm{s}$ et la longueur considérée, il se situe à :

- 3,90 marche normale:

- 5,20 en plus basses eaux de la mer et pertes de charges maximales.

La différence de cote entre ce plan d'eau et le niveau normal de la nappe peut avoir une incidence non négligeable sur le niveau de celle-ci au voisinage et sur sa salinité par pénétration d'eau venant de la mer.
L’écoulement permanent qui šétablira à long terme dans les talus de déblais est fonction de la perméabilité du sol: il sera nécessaire de toute façon de revêtir les talus d'une recharge drainante pour garantir leur stabilité.

\section{Ouvrage en tête des conduites}

Cet ouvrage a pour but dancrer les extrémités de conduites au niveau compatible avec le fonctionnement du système. Il isole en outre le chenal de la mer et doit permettre :

- de fermer une conduite pour révision de façon à contrôler le courant à l'intérieur;

- cette fermeture étant réalisée, de permettre d'introduire dans la conduite l'engin prévu pour leur nettoyage ou leur inspection.

L'ouvrage peut être constitué en béton ou par une digue: la construction en béton permet plus facilement l'aménagement d'une partie d'une crête déversante nécessaire en cas darrêt accidentel des groupes.

Sa réalisation nécessitera la mise hors d'eau du chantier côté mer et exécution de rabattement de nappe.

\section{Prise d'eau}

Elle doit se situer à une profondeur supérieure à deux fois le plus grand creux de houle $(-6,-0.50)$.

Laspiration proprement dite doit se trouver au-dessus d'une hauteur de 4.00 par rapport au fond.

Pour éviter de créer des courants verticaux qui "aspirent " les poissons, la prise d'eau se fait au travers d'une surface cylindrique verticale de hauteur $3,80 \mathrm{~m}$ utile. avec une surface brute déterminée en fonction d'une vitesse d'entrée voisine de $0.30 \mathrm{~m} / \mathrm{s}$

Ces différentes données correspondent à une cote de fond atteignant sensiblement $-15,00$, cote admise pour la première des études.

La figure 6 montre une réalisation possible de cette prise pour deux conduites jumelées $\emptyset 3,20 \mathrm{~m}$.

La conception semble peu influencée par la profondeur : pour les prises deau peu profondes, une superstructure émergente peut être envisagée : elle ne parait pas indispensable.

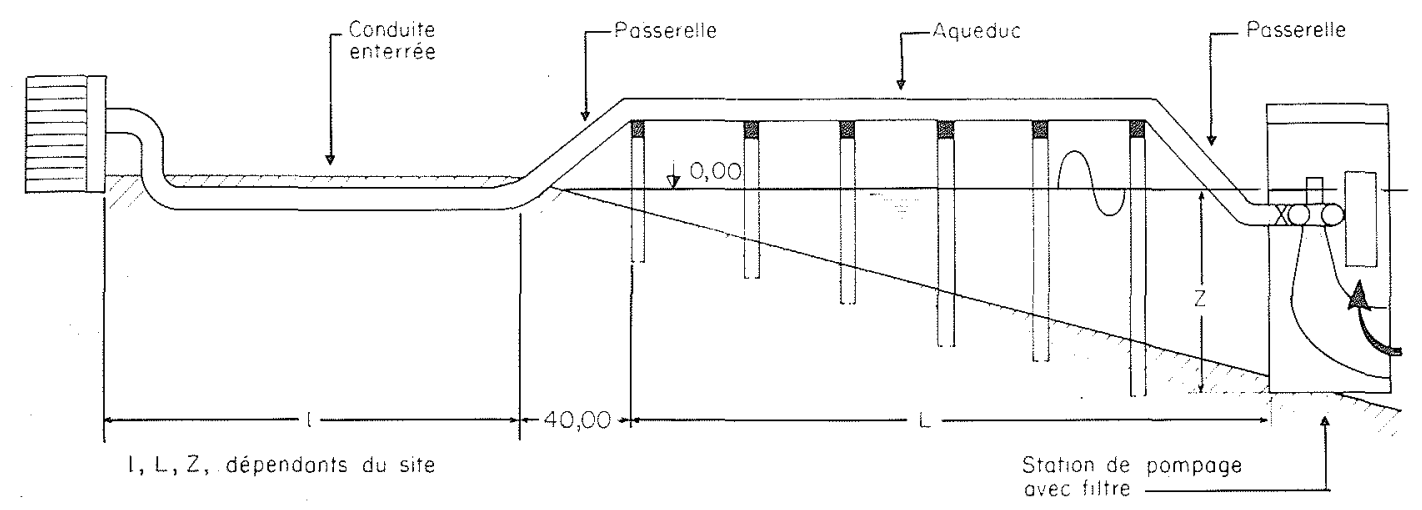

7 / Schéma B 
Elle pourrait être complétée si nécessaire par un dispositif de nettoyage du fond, à brancher sur une embarcation spécialisée dans l'entretien. Des bouées flottantes signaleront sa présence à la navigation.

\section{Amenées d'eau par conduites aériennes}

\section{PREMIER SCHÉMA TYPE}

Cette solution n'est réalisable qu'avec une station de pompage en mer.

Le schéma-type de solution est a priori le schéma B: fig. 7.

- Station de pompage en mer avec grilles d'entrée, batardage et filtres Beaudrey à tambour.

Des vannes à ouverture automatisée à la sortie des pompes permettent une mise en route progressive. L'étude de la station doit tenir compte des auxiliaires, locaux électriques et annexes.

- Conduites aériennes aboutissant au rivage.

- Conduites enterrées entre rivage et la centrale.

Ce schéma comporte des conduites pour le transport terrestre, car leur remplacement par un bassin nécessiterait un refiltrage des eaux avant les condenseurs.

La caractéristique de ce schéma est également que les circuits de chaque pompe sont indépendants - chaque unité nucléaire est alimentée par deux pompes - minimum nécessaire pour permettre l'entretien d'un des circuits.

Bien que la station de pompage soit en mer, la solution reste sûre : l'accès permanent à la centrale est assuré sans intervention de moyens maritimes.

\section{ÉLEMENTS COMMUNS \\ INDÉPENDANTS DE LA PROFONDEUR DE PRISE D'EAU - PONT AQUEDUC}

Les amenées d'eau provenant des deux pompes précitées ( 2 fois $22 \mathrm{~m}^{3} / \mathrm{s}$ ) sont jumelées et constituées par les évidements internes - de sections trapézoïdales - d'un pont caisson de hauteur constante, à âme centrale, formant pont aqueduc (voir figure).

L'importance de l'ouvrage (largeur $34 \mathrm{~m}$, longueur $2000 \mathrm{~m}$ environ) justifie l'acquisition d'un matériel de lancement de caractéristiques exceptionnelles, permettant la mise en place de tronçons de pont préfabriqués, pleine section, pouvant atteindre 700 à 750 tonnes.

Ce procédé porte le nom de "Système Macchi » du nom de son inventeur ( $\left.{ }^{1}\right)$, et implique la mise en place, au moyen d'une poutre de lancement d'éléments préfabriqués de deux types :

- les éléments d'appui, de type A, disposés symétriquement à cheval sur les piles et correspondant, sur toute leur longueur, aux 2/5 de la portée libre entre appuis;

- les éléments de travée, de type $T$, posés entre deux éléments de type $A$, et couvrant les $3 / 5$ de la portée.

(1) Développé en France par la Lilleroise de Constructions, sous licence Aspem-Pontes (Italie).
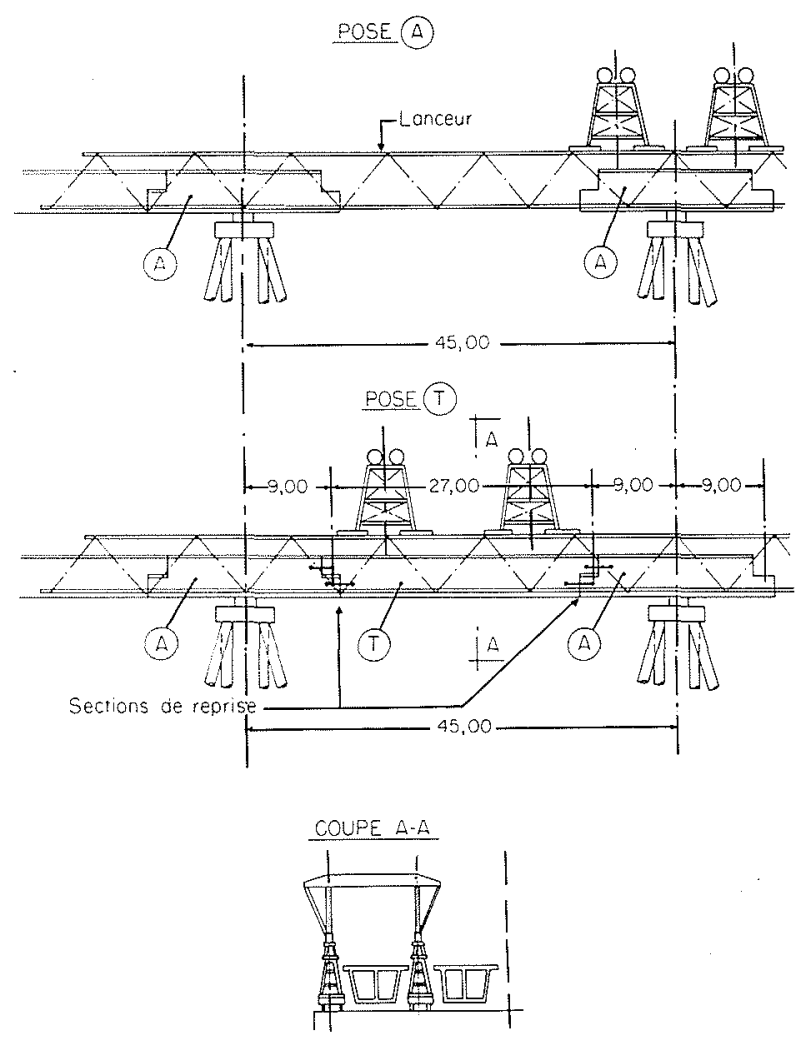

8 / Aqueduc

La portée entre appuis est de $45 \mathrm{~m}$. Les éléments $\mathrm{T}$ ont une longueur de $27 \mathrm{~m}$, leur poids est de $730 \mathrm{t}$ environ. Les éléments $A$ ont une longueur de $18 \mathrm{~m}$; ils présentent la particularité, par rapport aux poutres caissons classiques, d'une disposition "à l'extérieur " des raidisseurs sur appui, le libre passage de la veine fluide interne excluant toute possibilité de diaphragme.

Les éléments de type $A$ et de type $T$ sont liaisonnés entre eux par dés joints à durcissement accéléré soumis à une précontrainte par câbles courts.

\section{JONCTION DES ÉLÉMENTS}

Le procédé est caractérisé par la mise en cuvre de trois types de joints :

a) les joints assurant une jonction immédiatement définitive;

b) les joints réalisant en premier lieu une jonction temporaire et permettant une certaine adaptation de la structure : une intervention ultérieure permet de transformer ces joints provisoires en joints définitifs et d'assurer, en particulier, l'étanchéité requise pour le pont aqueduc; pour cette jonction définitive, des appuis en néoprène fretté entre élément $\mathrm{A}$ et $\mathrm{T}$ permettent d'obtenir un nivellement parfait ;

c) les joints permanents de dilatation.

Le problème d'étanchéité au droit de ces joints a été résolu pour des caissons de tunnel sous-marin, ce sont des joints pressurisés, vissables et éventuellement remplaçables. 


\section{DESCRIPTION}

\section{DU PROCÉDE DE CONSTRUCTION}

Le lanceur intéresse toujours les deux dernières travées, en prenant appui sur les trois piles correspondantes.

L'élément A est calé par les portiques du lanceur dans sa position définitive. de telle façon que son intrados vienne presque affleurer les faces supérieures des appareils d'appui. Le contact final est assuré par l'injection des résines epoxydiques faite à lintérieur du caisson. au travers de trous spéciaux ménagés à cet effet.

On procède alors à la pose de l'élément $T$ qui complète la travée, puis au clavage des joints et. la travée terminée. le cycle se répète jusquà la fin de la construction du pont aqueduc.

\section{PILES}

La solution retenue comporte des pieux métalliques foncés à une profondeur suffisante dans le sable: l'exécution est facilitée par l'emploi de plates-formes auto-élévatrices. Elle a été préférée aux autres solutions possibles : enceinte de palplanches qui s'accommode mal de brusques tempétes toujours à redouter, caissons havés ou pneumatiques.

Le lanceur des poutres peut d'ailleurs être adapté pour le battage des pieux.

\section{STATION DE POMPAGE (fond $-15,00$ )}

Pour des profondeurs telles que $-15,00$. la station de pompage a été conçue pour un matériel semblable à celui des installations terrestres correspondantes: pompes Bergeron. filtres Beaudrey à tambour.

Le projet complet comporte deux caissons correspondant chacun à deux tranches nucléaires et comportant donc chacun quatre pompes de $22 \mathrm{~m}^{3} / \mathrm{s}$.

Chaque caisson comporte deux rangées de six cellules identiques, encadrées par deux cellules d'extrémité plus vastes destinées a l'amortissement de la houle: leur dimension pourrait d'ailleurs s'avérer insuffisante.

La construction de caisson de cette importance. $90 \mathrm{~m} \times 35 \mathrm{~m}$. hauteur $25 \mathrm{~m}$. nécessite une souille de profondeur minimale de $6 \mathrm{~m}$, mais une profondeur plus importante serait nécessaire si on désire mettre à flot la structure après l'avoir montée à sa cote définitive.

A titre de comparaison on peut rappeler que des caissons de $30.5 \mathrm{~m} \times 38 \mathrm{~m}$ ont été réalisés pour la centrale d'Aramon, en caissons métalliques $10 \mathrm{~m} \times 38 \mathrm{~m}$ assemblés après flotlaison.

\section{CAS DE PRISE D'EAU EN PROFONDEUR IMPORTANTE}

Dans cette hypothèse. la station de pompage a été simplifiée par report des filtres Beaudrey en site terrestre, immédiatement à l'amont des condenseurs qui ne nécessitent qu'une charge d'eau peu importante (schéma B') : les pompes refoulent dans un bassin à niveau maintenu constant. à laval duquel les filtres travaillent dans les conditions habituelles.
Le problème soulevé alors est celui des protections nécessaires pour les pompes : le parti retenu comporte des grilles relevables extérieures, de façon à arrêter les paquets d'algues ou végétations similaires; la réalisation de grilles relevables pose toutefois des problèmes de fiabilité en milieu marin.

Le caisson, destiné à recevoir quatre pompes à aspiration verticale, comprend quatre cellules périphériques et deux cellules centrales de distribution des conduites et de locaux techniques nécessaires en partie supérieure: ses dimensions seraient denviron $36 \mathrm{~m} \times 47 \mathrm{~m}$. La construction serait à concevoir en deux phases du fait que le tirant d'eau du caisson terminé atteint $17 \mathrm{~m}$. Un ballastage assez complexe doit être étudié pour le stabiliser après sa mise en place.

\section{PLANNING}

Le planning serait conçu de façon que les éléments d'accès correspondant à un aqueduc soient en service pour l'achèvement de la station de pompage et les montages.

\section{Synthèse des conclusions}

Le point d'aboutissement des deux types de solution présentés est assez différent et fait l'objet des remarques suivantes qui serviront de conclusion à cette étude.
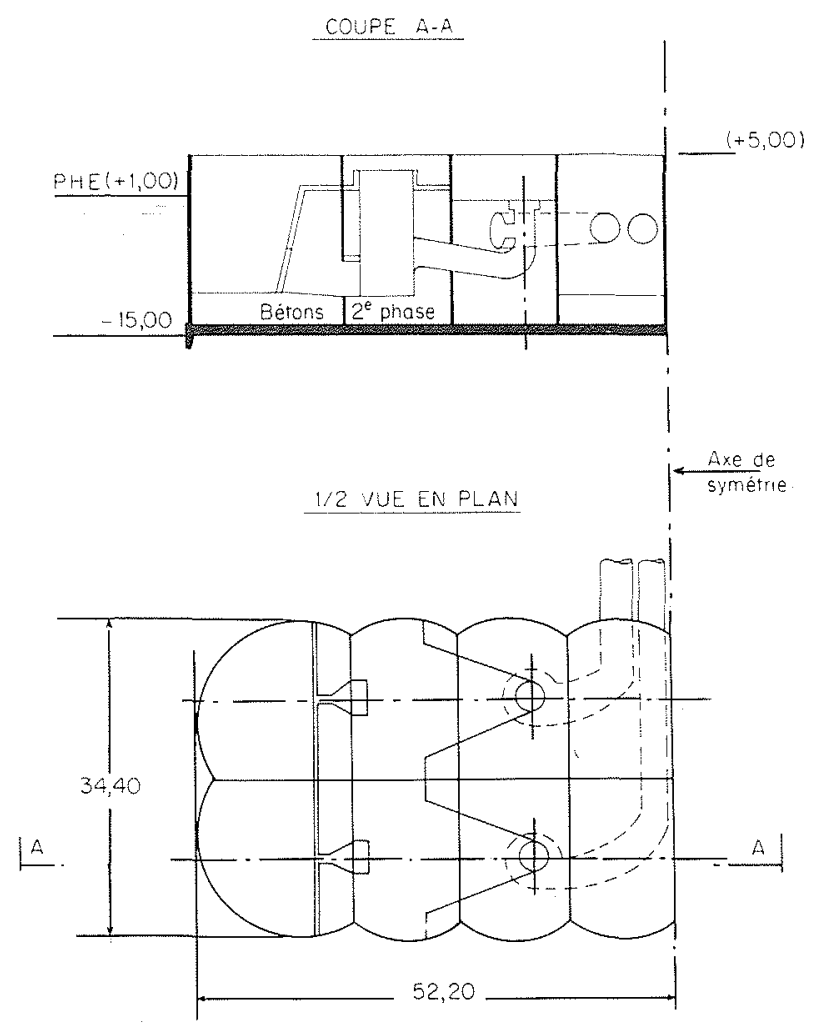

9 / Station de pompage $-15,00$ 


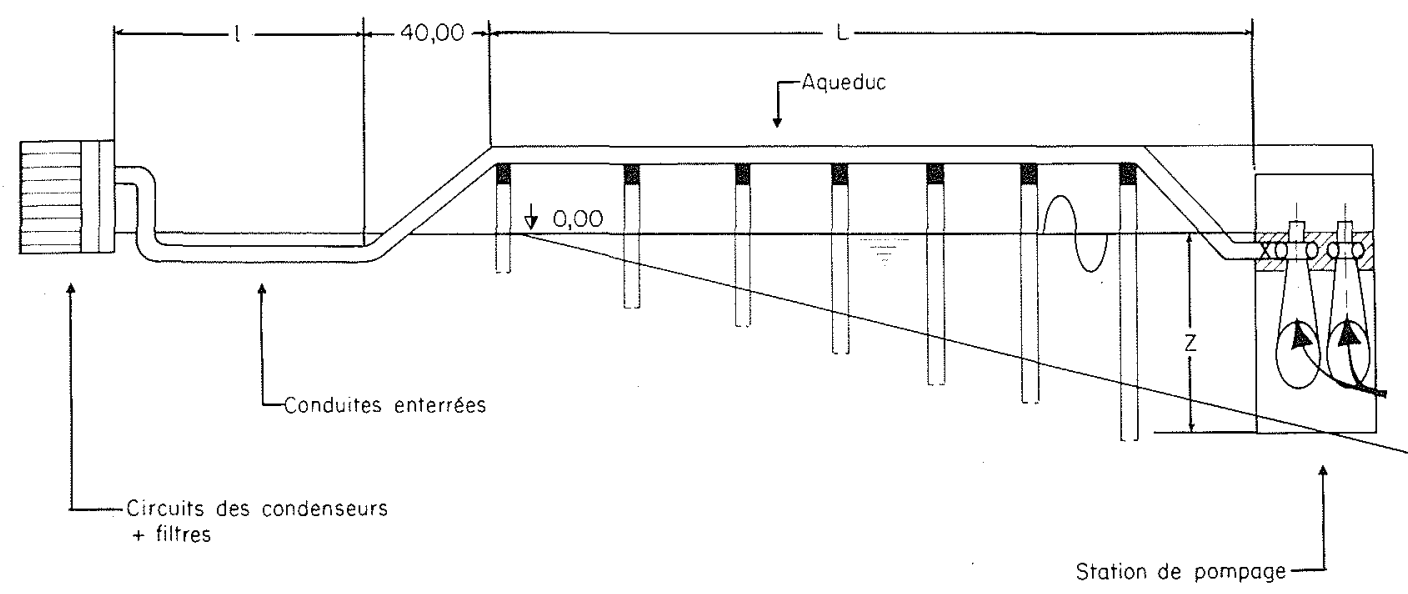

Variante: Ia conduite enterrée peut être remplacée par un bassin hout

$L, L, Z$, dépendent du site

10 / Schéma B'

\section{SOLUTION TYPE A}

On a le sentiment, après l'étude faite, que l'amenée d'eau par conduites immergées a pu être assez bien définie et qu'elle pourrait faire l'objet d'estimations sûres sur un site bien défini, en excluant toutefois le cas des fonds rocheux qui n'a pas été considéré dans le cadre de cette étude.

L'entretien des conduites peut être assuré par engin marinisé, ce qui permet de ne jamais vidanger les conduites et poser des conduites au large sans lestage.

L'économie maximale est obtenue en réduisant le nombre des conduites au minimum compatible avec une exploitation sûre, minimum qui correspond, pour le débit de $44 \mathrm{~m}^{3} / \mathrm{s}$ par tranche nucléaire, à une conduite d'un diamètre voisin de $4,00 \mathrm{~m}$. La pose de conduite de plus gros diamètre, qui pourrait présenter un intérêt économique pour le débit de refroidissement de $63 \mathrm{~m}^{3} / \mathrm{s}$, n'a pas été étudiée car il semble qu'une réalisation éventuelle de ce type de solution devrait commencer par l'emploi de tubes ne dépassant pas un diamètre de l'ordre de $4,00 \mathrm{~m}$.

On notera en outre que, contrairement à ce qu'on pouvait attendre - mais est-ce une conclusion définitive? l'incidence de la profondeur de prise n'est pas très importante.

\section{SOLUTION TYPE B}

Le schéma général de ces solutions est plus complexe et a donné lieu à l'examen de plusieurs variantes. On peut retenir :

- une grande incidence de la profondeur sur l'économie du système;

- la nécessité, de réduire la longueur des conduites en aqueduc au maximum, par exemple en utilisant des conduites enterrées ou immergées à faible distance du rivage:

- la possibilité éventuelle de réduire le coût de ce type de prise d'eau en prévoyant un bassin à niveau élevé dès que les fonds marins et l'environnement le rendent possible; la conséquence en est le report des filtres Beaudrey au voisinage de la centrale, ce qui réduit les dimensions de la station de pompage elle-même, réduction presque impérative pour des fonds atteignant ou dépassant -25, .

- l'intérêt d'utiliser l'aqueduc réalisé à des fins touristiques ou portuaires: on dispose en effet d'une plateforme très large permettant d'accéder à des fonds importants: il serait possible de la compléter par un apontement permettant l'accès de navires marchands. 


\section{Discussion}

Président : M. J.-C. LEBRETON

M. le Président remercie vivement M. Collange de son exposé parfois agréablement teinté d'un certain futurisme.

En raison de l'heure tardive, il propose de lever la séance dont il tire la conclusion en ces termes:

Les trois exposés de la matinée, consacrés à un sujet particulièrement important et en pleine évolution, ont été. très intéressants et fort suggestifs.

Je forme le vœu que nous nous retrouvions, à nouveau - dans un an environ - à la Société Hydrotechnique de France pour faire le point sur ce thème qui - bien que déjà plusieurs fois traité à cette tribune - est loin d'être entièrement exploré.

Je souhaiterai que soient traités, non seulement l'aspect architecture des circuits d'eau mais aussi la technologie des prises et aqueducs eu égard aux problèmes écologiques qui peuvent se présenter; l'aspiration des méduses - constatée à la centrale suédoise de Ringhals - et peut être des poissons pourrait se produire dans d'autres centrales; il est nécessaire d'y pallier, tant pour la conservation de la vie marine que pour éviter les indisponibilités onéreuses qu'elle pourrait imposer à un outil de production dont la fiabilité doit être quasi-absolue.

La séance est levée à 12 h 40 . 